REVUES CRITIQUES

\title{
HISTOIRE DU JAPON
}

Le Sansom* est un classique des études japonaises. Certes, la page de garde précise que la traduction en langue française a été effectuée à partir de l'édition anglaise publiée en 1974. Mais il faut savoir qu'en fait la version originale de l'ouvrage remonte à une génération puisque la publication s'est échelonnée en trois volumes, de 1958 à 1964 . Ce livre a couronné une longue carrière de chercheur dont les étapes ont notamment été marquées en 1950 par The Western World and Japan et, dès 1931, par Japan. A Short Cultural History (traduction française publiée par Payot). George Sansom est mort en 1965.

Hélas, cette monumentale synthèse de l'histoire japonaise des origines à 1867 - c'est-à-dire le moment où le Japon, sortant de son isolement, s'ouvre à l'Occident et entre dans l'ère Meiji qui fonde le Japon moderne - est une œuvre non seulement ancienne par sa publication (près de trente ans pour les premiers chapitres) mais souvent archaïque et dépassée. Cela tient certes aux innombrables découvertes, recherches et mises au point qui ont eu lieu entre-temps mais surtout à la façon de l'auteur de concevoir l'histoire. Le livre pèche essentiellement par ses carences et erreurs méthodologiques.

L'érudition, la maîtrise parfaite des sources et de la langue, l'intelligence de l'auteur ne sont absolument pas en cause. Pas plus que l'ampleur du panorama qu'il nous offre sur le Japon : tableaux chronologiques, généalogies, politique intérieure, affaires étrangères (p. 728), études toponymiques (p. 696), éducation (avec un appendice, p. 959, sur l'éducation supérieure), religion (chapitre LIV essentiellement consacré au confucianisme; mouvements antichrétiens étudiés p. 790-795), culture, littérature, institutions, vie de cour, vie militaire, vie quotidienne à la ville comme à la campagne, économie qui ne néglige ni les finances ni le commerce extérieur, bref rien ne semble manquer.

Pourtant tout cela est comme saupoudré et finalement noyé dans un récit totalement événementiel sans que jamais - par exemple sur le bouddhisme, fondamental dans l'histoire du Japon - on ne puisse saisir le moindre fil conducteur,

\footnotetext{
* George Sansom, Histoire du Japon. Des origines aux débuts du Japon moderne. Trad. de l'anglais d'Eric Diacon, Paris, Fayard, 1988, 16,5 × 24, 1020 p., postface, bibliogr., glossaire, index.
}

Revue de synthèse : IV S. N ${ }^{0} 1$, janv.-mars 1991. 
la moindre explication susceptible, au-delà de l'anecdote, de démontrer les causalités, démonter les mécanismes et finalement susciter, qu'on soit spécialiste du Japon ou simple curieux de l'histoire non occidentale, la réflexion.

La postface de Danielle Eliseeff, incomparable experte de l'histoire japonaise, nous offre en deux pages une mise au point dont la justesse n'étonnera point :

“ Le livre refermé, il reste une impression ineffaçable : celle d'un style et, à travers le style, la présence d'un homme au ton inimitable [...] Son texte, résolument événementiel, s'inscrit dans une tradition d'écriture narrative, linéaire que l'historiographie moderne a enrichie, depuis, de points de vue thématiques ; c'est l'ossature de l'histoire telle qu'on l'apprenait à l'école dans les manuels d'autrefois. Elle permet de mieux apprécier, par comparaison, combien les dépouillements d'archives, l'archéologie, la linguistique, l'anthropologie ont apporté depuis trente ans de jalons et d'éclairages précieux sur l'évolution du Japon ancien. "

C'est avouer, chose rarissime dans la présentation d'un livre, que ce livre a beau être un "monument ", c'est un monument dépassé. C'est pourquoi sans doute l'éditeur a préféré faire passer cette présentation à la fin, sous forme de postface, au lieu de le placer, ce qui eût semblé plus logique mais n'eût pas manqué de résonner comme une mise en garde dissuasive, en tête de l'ouvrage sous forme de préface.

De toute évidence, sauf à saboter délibérément le projet de l'éditeur, Danielle Eliseeff ne pouvait souligner ce qui est le plus gênant dans le livre de Sansom, en dehors du côté systématiquement événementiel, et qui tient à la méthodologie même et à la conception de l'historien formé dans la tradition de l'ethnologie à la Frazer.

Rappelons que l'auteur du Rameau d'or, livre contesté en France dès sa parution et relégué au rang des curiosités pour savant en chambre - Frazer ne connaît comme terrain que sa bibliothèque - par Georges Dumézil ${ }^{1}$, a utilisé

" sans aucune discrimination des documents recueillis par les premiers ethnographes chez les populations dites primitives, ceux collectés par les folkloristes dans les sociétés européennes traditionnelles et les documents provenant des textes, des cultures de l'Antiquité classique et de l'Asie Mineure, textes d'une part lacunaires en raison des hasards qui ont présidé à leur conservation, alors que beaucoup d'autres ne sont pas parvenus jusqu'à nous, et d'autre part hétérogènes quant à leur destination " ${ }^{2}$.

Le tout sans aucune critique des sources dans un bric-à-brac qui ne peut même pas être utilisé pour ce qu'on appellerait aujourd'hui l'élaboration d'une « banque de données $»$.

Dans la lignée de Frazer, devenu héros anobli de la science anglaise, pour qui la méthode se résumait en l'accumulation et le fourre-tout, la mise à la suite sans aucun sens critique des mythes et des rituels les plus dissemblables et les plus

1. Georges DumEzIl, "Comparative Study of the Religion of the Indo-European Peoples ", Criterion, Automn 1969. Voir aussi Claude Lévi-Strauss, Du miel aux cendres, Paris, Plon, 1966.

2. Nicole Belmont, Michel Izard, introduction à la rééd. du Rameau d'or en français, 4 vols, Paris, Robert Laffont, «Bouquins», 1981, t. 1, p. XXVII. 
éloignés géographiquement et dans la diachronie ${ }^{3}$, Sansom compare sans cesse, de façon lassante et finalement irritante, le moindre événement de l'histoire japonaise ancienne avec les civilisations anciennes occidentales.

Ainsi, page 31 , où l'auteur, notant que « le sang versé est polluant », ajoute :

«Le mot Kega est le mot original pour souillure et dans le langage actuel les mots Kegare, Kegare-ru et Kegasu signifient tache, être taché et tacher. Il y a une proche analogie avec les idées de pureté dans la Grèce antique, bien qu'à Athènes l'eau ne suffit pas toujours à laver le sang versé et qu'il fallüt parfois utiliser le sang d'animaux sacrificiels. Selon la conception grecque telle qu'elle s'exprime dans l'Orestie d'Eschyle, seule le sang pouvait laver le sang. "

Et de conclure par une formule qui montre la vacuité de ce qui précède : « Il n'y a rien de comparable dans les idées du Japon primitif. " Pourquoi d'ailleurs ne pas comparer avec les interdits du Talmud, la conception de la souillure par le sang dans plusieurs centaines de castes hindoues, ou encore Don Quijote de la $M a n c h a^{4}$, puisqu'on en est à citer ce qui passe par la tête, au crible des souvenirs universitaires de l'auteur?

Ainsi encore, page 101, à propos du Kampaku, ministre qui, porte-parole et conseiller du Trône, détint en fait la réalité du pouvoir à partir de 880 , Sansom enchaîne naturellement : "Cette charge irrégulière n'est d'ailleurs pas sans parallèles. En Europe, les maires des palais mérovingiens et en Asie les Ranas du Népal ressemblent fort aux régents Fujiwara qui usurpèrent pratiquement la prérogative royale. " $\mathrm{Et}$, derechef, la note qui annule le propos : « Il y a toutefois une différence considérable. Tandis que...»

De même, page 30 , où l'auteur nous sert un parallèle fumeux entre la mythologie grecque et romaine véhiculée et conservée en Occident par le christianisme et les mythes des dieux locaux japonais véhiculés et conservés par le bouddhisme. Comme soudain lucide, il conclut à nouveau par : " Il faut toutefois admettre que le parallèle est inexact; en effet, ..."

Il y a plus grave, lorsque, page 26, Sansom établit un parallèle entre numen ${ }^{5}$ et mana ${ }^{6}$ qu'il rapproche du Kami japonais en posant le principe d'une équation parfaite entre les trois termes, il use de notions totalement périmées et dont la fausseté dans leur application à l'histoire des religions avait largement été démon-

3. Cette méthode n'a rien à voir avec l'analyse dite "cross-cultural " qui, dans l'anthropologie contemporaine, "vise à établir sur un nombre aussi grand que possible de sociétés, des corrélations entre les phénomènes culturels " pour reprendre, précisément, la définition d'une spécialiste de terrain actuelle du monde japonais, Liza Crinfield Dalby, in Geisha, trad. franç., Paris, Payot, 1985, p. 13.

4. La Mancha désigne en espagnol une province, célèbre par ses moulins à vent, ou la tache, la souillure (du latin maculare).

5. Numen, que Sansom ne définit d'ailleurs pas, désigne en latin la volonté divine puis la puissance divine. Le mot vient du verbe nuo que l'on trouve surtout dans le substantif nutus (signe de tête). Cf. A. Ernout, A. Meillet, Dictionnaire étymologique de la langue latine, Paris, Klincksieck, $4^{\mathrm{e}}$ éd., 1967, p. 452.

6. Mana, mot mélanésien désignant une force surnaturelle, indifférenciée et impersonnelle, présente notamment dans les objets, dont on a voulu à tort faire un équivalent de numen. 
trée au moment où l'auteur publie son livre. Voir dans le numen romain une puissance divine qui, comme la mana, peuplerait la nature, le monde animal et l'être humain lui-même, relève de fantasmes ou de fantaisies qui remontent à la fin du $\mathrm{XIX}^{\mathrm{e}}$ siècle et au début du $\mathrm{xx}^{\mathrm{e}}$.

Que dire, enfin, de la comparaison saugrenue entre le mythe d'Izanagri parti à la recherche de son épouse Izanami et l'histoire d'Orphée et d'Eurydice ? Saugrenue non point cette fois parce que la comparaison met en parallèle un mythe japonais et un mythe grec - on est ici dans le cas d'archétypes sans doute universels - mais parce que la comparaison tourne rapidement en un désolant jugement de valeur européanocentrique qui considère le récit mythique japonais comme " prosaïque " au regard d'un Milton que Sansom cite avec complaisance avant de conclure, page 30 :

« Il semblerait que l'impuissance des Japonais à développer la tragédie du mythe de Perséphone (ou d'Eurydice) témoigne d'une différence essentielle de vision. Pendant des siècles, ces thèmes classiques (et des thèmes similaires tires de la Bible) ont en Europe nourri la tragédie. Mais l'imagination des Japonais ne parait pas émue de la même manière, même par des histoires qui, comme celle-ci, appartiennent à leur tradition nationale."

"Ah, ces jaunes!", serait-on tenté de dire au terme de ce chapitre...

Cela ne doit pas sembler anecdotique. Ressortissant totalement de la méthode Frazer qui combine la juxtaposition non critique au jugement de valeur hypercritique pour tout ce qui n'est pas accidentel, Sansom, comme l'illustre auteur du Rameau d'or, massacre ce qu'il compare ou ce qu'il découvre. Il est un bon représentant de cette époque où les archéologues recherchaient la belle pièce, la trouvaille, et massacraient l'environnement des objets, rendant ainsi l'exploitation historique, culturelle ou autre souvent problématique. Comme l'écrit un excellent spécialiste de l'Asie et singulièrement du Japon ${ }^{7}$, Louis Frédéric, dans son Manuel pratique d'archéologie (Paris, Robert Laffont, $2^{e}$ édition, 1967, p. 12-13) :

"La recherche de la " belle pièce ", l'appât du gain, le goût de la renommée furent les causes de ces catastrophiques bouleversements de terre [...]. Un objet quelconque, indépendamment de sa valeur artistique, ne " parle " pas à l'archéologue s'il est séparé du contexte dans lequel il a été trouvé."

L'un et l'autre défaut, celui des historiens et ethnologues qui consistait à mettre en parallèle les sociétés et civilisations les plus dissemblables et les plus éloignées dans le temps, et celui des archéologues qui consistait à additionner de façon antihistorique des pièces monnayables et montrables comme chefs-d'œuvre dans les musées, ces deux défauts, pour antinomiques qu'ils puissent paraître, relèvent d'une même erreur : privilégier une " approche culturaliste », aussi envahissante que mystifiante. Cette dernière formule est employée par Philippe Pons dans le très sévère compte rendu du livre de Sansom dans le Monde des livres du 3 mars 1989.

7. Louis FrédérIC, La Vie quotidienne au Japon à l'époque des Samourai, 1185-1603, Paris, Hachette, 1969, et ID., Le Shintô, esprit et religion du Japon, Paris, Bordas, 1972. 
A sa suite, nous poserons la question : traduire, au bout de trente ans, un tel ouvrage en français - et le vendre à un prix aussi élevé - a-t-il un sens ?

Le grand public, à l'évidence, le boudera ou, si d'aventure, il l'achète ou l'emprunte en bibliothèque, il n'ira pas jusqu'au bout. L'étudiant en japonais ou le spécialiste qui, par la force des choses, savent l'anglais, liront l'édition originale, si besoin est, mais recourront largement aux nombreux ouvrages excellents parus ces dernières années sur le Japon ${ }^{8}$.

Le danger d'une telle édition nous semble essentiellement concerner la troisième catégorie de lecteurs potentiels, la plus vaste en fait : les étudiants, enseignants non versés dans l'étude du Japon, lisant le Sansom in extenso et s'imprégnant des idées et d'une méthode surannées et, tout compte fait, dangereuses. Dès lors, pour inverser la fameuse formule de Boulay de la Meurthe, attribuée à tort à Talleyrand, la publication de ce livre serait pis qu'une faute, un crime...

Alain HouLou.

8. A l'intention des étudiants et chercheurs dans le domaine japonais, l'« École des langues orientales " (Paris III) a publié sous la plume de Francine Héralt, d'irremplaçables Éléments de bibliographie japonaise permettant de faire le point récent sur les diverses questions et, notamment, sur l'histoire du Japon. Notons que la bibliographie japonaise en langue française s'est récemment enrichie de quatre titres : L'Etat du Japon et de ses habitants, dir. Jean-François Sabouret, Paris, La Découverte, 1988 ; Les Bases de la puissance du Japon, dir. Jean Esmein, Paris, Fondation pour les Études de défense nationale, 1988 ; Robert Guillain, Les Geishas ou le monde des fleurs et des saules, Paris, Arléa, diff. Seuil, 1988 ; enfin, pour une excellente mise au point sur la préhistoire et l'histoire générale du Japon, le Cultural Atlas of Japan de Martin Collcutr, Marius JANSEN, Isao KumakurA, Oxford, Equinox, 1988, trad. franç. par L. FréterIC, Paris, Éd. du Fanal, 1989. 